\title{
Structural, Optical, Morphological and Dielectric Properties of Cerium Oxide Nanoparticles
}

\author{
Devadoss Mangalam Durai Manoharadoss Prabaharan ${ }^{*}$, Karuppasamy Sadaiyandi ${ }^{b}$,
}

\author{
Manickam Mahendranc, Suresh Sagadevan ${ }^{d}$
}

${ }^{a}$ Department of Physics, NPR College of Engineering and Technology,

Natham, Dindigul, Tamil Nadu, India

${ }^{b}$ Department of Physics, Alagappa Government Arts College, Karaikudi, Sivaganga, Tamil Nadu, India

'Department of Physics, Thiagarajar College of Engineering, Madurai, Tamil Nadu, India

${ }^{d}$ Department of Physics, AMET University, Chennai-603 112, India

Received: November 18, 2015; Revised: January 12, 2016; Accepted: February 9, 2016

\begin{abstract}
Cerium oxide $\left(\mathrm{CeO}_{2}\right)$ nanoparticles were prepared by the precipitation method. The average crystallite size of cerium oxide nanoparticles was calculated from the X-ray diffraction (XRD) pattern and found to be $11 \mathrm{~nm}$. The FT-IR spectrum clearly indicated the strong presence of cerium oxide nanoparticles. Raman spectrum confirmed the cubic nature of the cerium oxide nanoparticles. The Scanning Electron Microscopy (SEM) analysis showed that the nanoparticles agglomerated forming spherical-shaped particles. The Transmission Electron Microscopic (TEM) analysis confirmed the prepared cerium oxide nanoparticles with the particle size being found to be $16 \mathrm{~nm}$. The optical absorption spectrum showed a blue shift by the cerium oxide nanoparticles due to the quantum confinement effect. The dielectric properties of cerium oxide nanoparticles were studied for different frequencies at different temperatures. The dielectric constant and the dielectric loss of the cerium oxide nanoparticles decreased with increase in frequency. The AC electrical conductivity study revealed that the conduction depended on both the frequency and the temperature.
\end{abstract}

Keywords: Cerium oxide, XRD, FTIR, FT-Raman, SEM, TEM, Dielectric studies

\section{Introduction}

Nanoscience deals with matter at nanoscale dimension, typically in the size range between $1 \mathrm{~nm}$ and $100 \mathrm{~nm}$. Unique phenomena/novel properties that are found in materials at the nanoscale enable nanotechnology to be applied in various fields and form a solid basis towards technological breakthrough. Interestingly, such properties are not observed at the atomic/molecular scale or at the bulk scale, thus making nanotechnology an emerging technology of the $21^{\text {st }}$ century. The surface to volume ratio is much higher in nanoparticles compared with their respective bulk materials. Therefore, surface atoms that are known to be in an energetic state different from that of the bulk atoms make significant contribution to the total free energy. Cerium oxide $\left(\mathrm{CeO}_{2}\right)$ is a semiconductor with wide band gap energy $(3.19 \mathrm{eV})$. In the recent years, much effort has been made towards the development of new synthetic routes for preparing nanostructure cerium oxides due to their potential uses in many applications, such as high-storage capacitor devices, buffer layers for conductors, fuel cells, polishing materials, UV blocks and optical devices ${ }^{1-7}$. The cerium oxide nanoparticles are prepared by various methods ${ }^{8-12}$. This paper deals with the preparation of cerium oxide nanoparticles using the precipitation method. The prepared nanoparticles were

*e-mail: duraiphysics2011@gmail.com characterized by powder X-ray diffraction analysis, FTIR, Raman spectrum, Scanning Electron Microscopy (SEM), Transmission Electron Microscope (TEM) UV-analysis and dielectric studies.

\section{Experimental Procedure}

Cerium oxide $\left(\mathrm{CeO}_{2}\right)$ nanoparticles were prepared by the precipitation method using cerium sulfate $\left(\mathrm{Ce}_{2}\left(\mathrm{SO}_{4}\right)_{2}\right)$, oxalic acid $\left(\mathrm{H}_{2} \mathrm{C}_{2} \mathrm{O}_{4}\right)$. In a typical synthesis procedure, $1 \mathrm{~g}$ of cerium sulfate dispersing was put into $100 \mathrm{~mL}$ distilled water to form a clear solution, which was stirred strongly at room temperature for about $5 \mathrm{~h}$. The obtained clear solution was added drop-wise to $10 \mathrm{ml}$ ammonia water under constant stirring condition for $2 \mathrm{~h}$. The resultant synthesis precipitate was washed with deionized water and dried at $70^{\circ} \mathrm{C}$ for $24 \mathrm{~h}$. The X-ray diffraction analysis (XRD) pattern of the $\mathrm{CeO}_{2}$ nanoparticles was recorded by using a powder X-ray diffractometer (Schimadzu model: XRD 6000 using $\mathrm{CuK} \alpha$ $(\lambda=0.154 \mathrm{~nm}))$ radiation, with a diffraction angle between 20 and $80^{\circ}$. The crystallite size was determined from the broadenings of corresponding X-ray spectral peaks by using Scherer's formula. The FTIR spectrum of the $\mathrm{CeO}_{2}$ nanoparticles was taken using an FTIR model Bruker IFS 66W Spectrometer. Raman spectrum was obtained using a 
Bruker RFS 27: stand-alone model Raman spectrometer. Scanning Electron Microscopy (SEM) studies were carried out on JEOL, JSM- 67001. Transmission Electron Microscope (TEM) image was taken using an H-800 TEM (Hitachi, Japan) with an accelerating voltage of $100 \mathrm{kV}$. UV-Vis absorption spectrum for the $\mathrm{CeO}_{2}$ nanoparticles was recorded using a Varian Cary 5E spectrophotometer in the range of 300-800 nm. The dielectric constant and the dielectric loss of the pellets of $\mathrm{CeO}_{2}$ nanoparticles in disk form were studied at different temperature using a HIOKI 3532-50 LCR HITESTER in the frequency range of $50 \mathrm{~Hz}$ to $5 \mathrm{MHz}$.

\section{RESULTS AND DISCUSSION}

\subsection{XRD analysis}

Structural identification of cerium oxide nanoparticles was done by means of X-ray diffraction in the range of angle $2 \theta$ between $20^{\circ}$ and $80^{\circ}$ as shown in Fig. 1. The excellent peaks (111), (200), (220), (311), (222), (400), (331), and (422) were obtained. It was clearly indicated that the fine crystalline and single phase of $\mathrm{CeO}_{2}$, could be indexed to the cubic structure. The broadened peak showed the nanometer-sized crystallites. The average nano-crystalline size (D) was calculated using the Scherrer formula,

$D=\frac{0.9 \lambda}{\beta \cos \theta}$

where $\lambda$ is the $\mathrm{X}$-ray wavelength, $\theta$ is the Bragg diffraction angle, and $\beta$ is the full width half maximum (FWHM) of the XRD peak appearing at the diffraction angle $\theta$. The average crystalline size was calculated from X-ray line broadening using Scherrer equation and it was found to be about $11 \mathrm{~nm}$.

\subsection{FTIR and FT-Raman Analysis}

The FTIR spectrum of the cerium oxide nanoparticles are shown in Fig.2. The spectrum clearly shows the three intense peaks situated at $3418,1612 \mathrm{~cm}^{-1}$ and below $700 \mathrm{~cm}^{-1}$. The weak absorption peaks at 2380 and $1430 \mathrm{~cm}^{-1}$ are attributed to the bending vibration of $\mathrm{C}-\mathrm{H}$ bands of the incorporated surfactant residuals. Furthermore, the O-C-O stretching band is also observed in the region $1300-1600 \mathrm{~cm}^{-1}$. The absorption band around $1634 \mathrm{~cm}^{-1}$ is attributed to the bending vibration of absorbed molecular water, which can be seen in all the specimens. The peak at $450-550 \mathrm{~cm}^{-1}$ is attributed to the $\mathrm{O}-\mathrm{Ce}-\mathrm{O}$ stretching mode of vibration.

Fig. 3 shows the Raman spectrum of cerium oxide nanoparticles. Ceria, which are cubic fluorite structure-metal dioxides, have a single Raman mode at $464 \mathrm{~cm}^{-1}{ }^{13}$. This peak confirms the cubic nature of the cerium oxide, which has $\mathrm{F}_{2 \mathrm{~g}}$ symmetry and can be viewed as a symmetric breathing mode of $\mathrm{O}$ atoms around each cation. As only the $\mathrm{O}$ atoms move, the vibrational mode is nearly independent of cation mass.

\subsection{SEM analysis}

Scanning Electron Microscopy (SEM) provided further approaching into the morphology and size details of the nanoparticles. The surface morphology of the prepared cerium oxide nanopartices was revealed through the SEM image as shown in Fig.4. It is clearly shown that the particles are formed in a spherical shape.

\subsection{TEM analysis}

TEM is commonly used for imaging and analytical characterization of the nanoparticles to assess the shape, size, and morphology. TEM images of the cerium oxide nanoparticles are shown in Fig.5. It is evident from the Fig. 5 that the particles were smaller in size and uniform in shape. The estimated particle size was around $16 \mathrm{~nm}$ with a narrow size distribution.

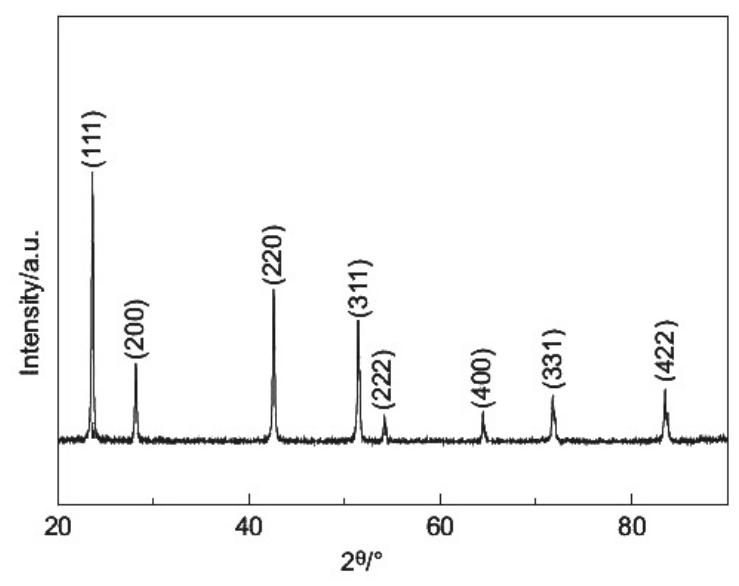

Fig.1. XRD pattern of nanoparticles

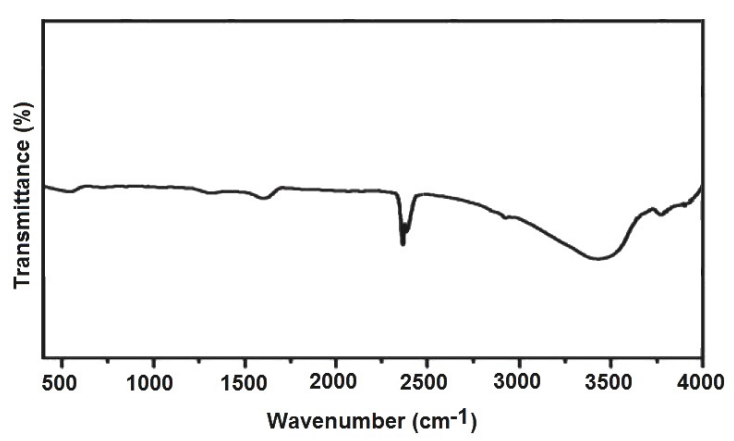

Fig.2. FT-IR spectrum of cerium oxide nanoparticles

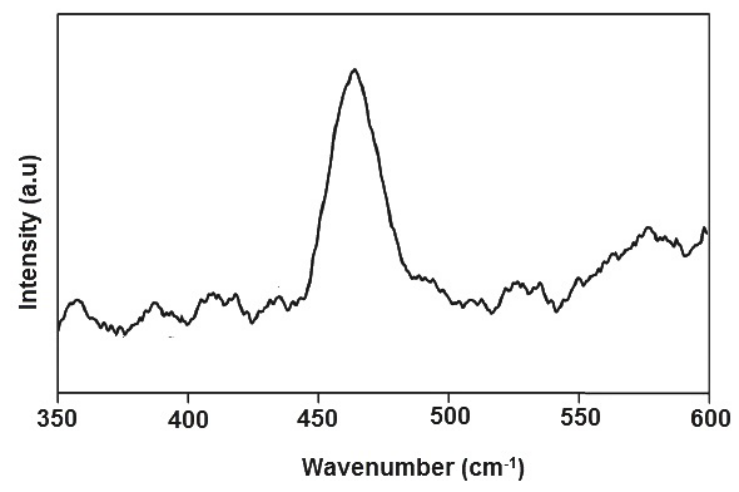

Fig.3. Raman spectrum of cerium oxide nanoparticles 


\subsection{UV analysis}

Optical absorption measurement was carried out on cerium oxide nanoparticles. Fig. 6 shows the variation of the optical absorbance with the wavelength of the nanoparticles.

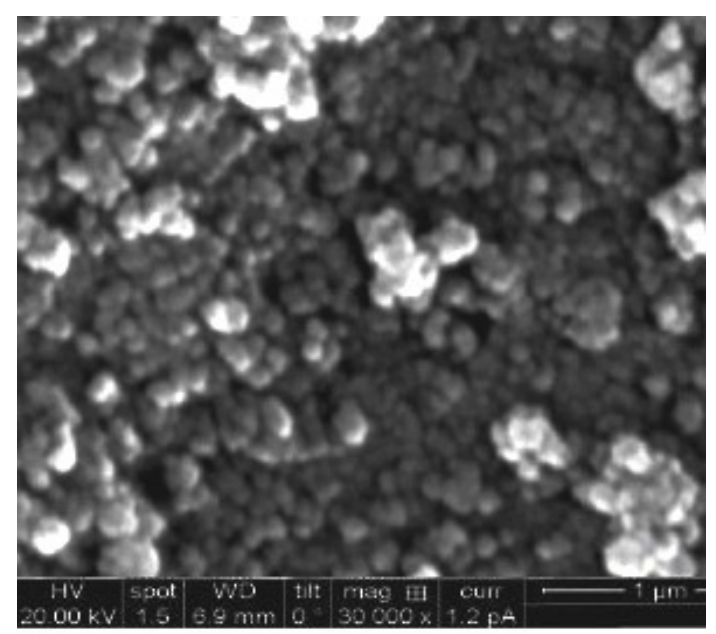

Fig.4. SEM image of cerium oxide nanoparticles

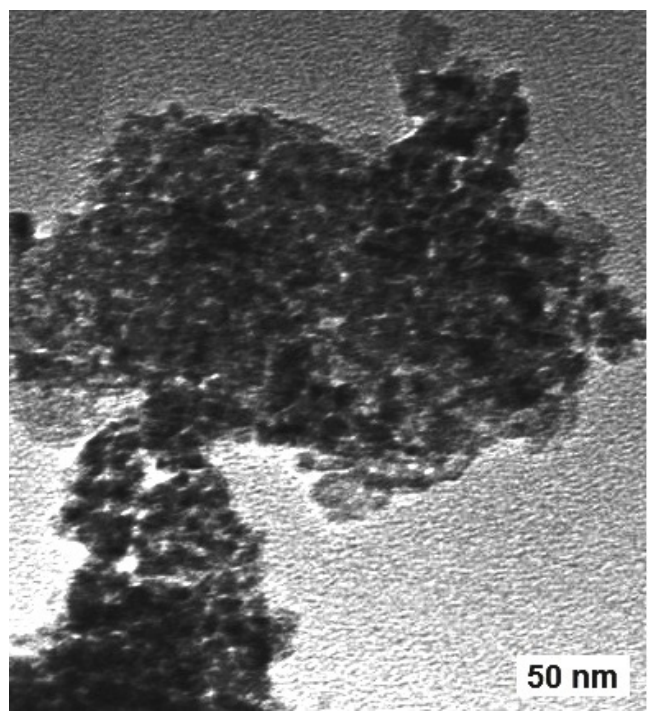

Fig.5. TEM image of cerium oxide nanoparticles

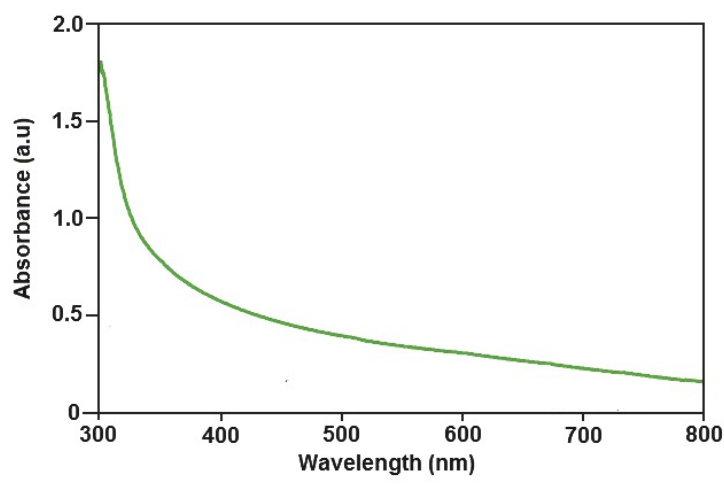

Fig.6. Optical absorption spectrum of $\mathrm{CeO}_{2}$ nanoparticles
The optical absorption coefficient was calculated in the wavelength range of $300-800 \mathrm{~nm}$. The UV-Vis absorption spectrum reveals that the absorption threshold edge was found to be $421 \mathrm{~nm}$. The spectra show a strong red shift of the absorption threshold edge compared with bulk $\mathrm{CeO}_{2}$ material $\left(\mathrm{E}_{\mathrm{g}}=3.19 \mathrm{eV}\right)$.

The optical absorption coefficient $(\alpha)$ was calculated from transmittance using the following relation

$\alpha=\frac{1}{d} \log \left(\frac{1}{T}\right)$

where $\mathrm{T}$ is the transmittance and $\mathrm{d}$ is the thickness. The study has an absorption coefficient $(\alpha)$ obeying the following relation for high photon energies $(h v)$

$\alpha=\frac{A\left(h v-E_{g}\right)^{1 / 2}}{h v}$

where $\alpha, \mathrm{E}_{g}$ and $A$ are the absorption coefficient, band gap, and constant respectively. The fundamental absorption corresponding to the optical transition of the electrons from the valence band to the conduction band can be used to determine the nature and value of the optical band gap $E_{g}$ of the nanoparticles. A plot of $(\alpha h v)^{2} v s(h v)$ is used to evaluate the direct optical band gap of the material and is shown in Fig.7. The band gap is determined by extrapolating the linear portion of the curve. The band gap was found to be $2.8 \mathrm{eV}$.

\subsection{Dielectric Properties}

The dielectric constant and the dielectric loss of the pellets of cerium oxide nanoparticles in disk form were studied for different frequencies and different temperatures. The dielectric constant is evaluated using the relation,

$\varepsilon_{r}=\frac{C d}{\varepsilon_{0} A}$

where $d$ is the thickness of the sample and A, is the area of sample. The variations of dielectric constant with frequency and temperature for cerium oxide nanoparticles are shown in Fig.8. The dielectric constant of cerium oxide nanoparticles is high at low frequencies and decreases rapidly with the applied frequency at all temperatures. The high values of the dielectric constant in the present study may be attributed to the increased ion jump orientation effect and the increased space charge effect exhibited by nanoparticles. Most of the atoms in the nanocrystalline materials reside in grain boundaries, which become electrically active as a

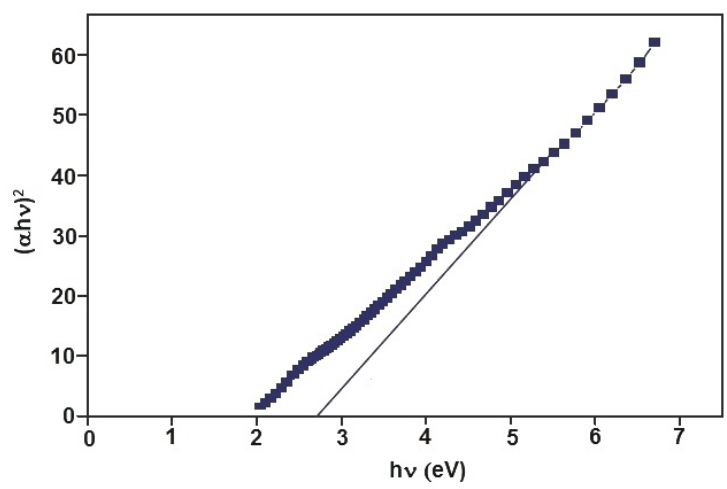

Fig.7. Plot of $(\alpha h v)^{2}$ Vs photon energy 
result of charge trapping ${ }^{14}$. The dipole moment can easily follow the changes in the electric field, especially at low frequencies. Hence, the contributions to dielectric constant increase through space charge polarization and rotation polarization, which occur mainly in interfaces ${ }^{15}$. Therefore dielectric constant of nanostructured materials should be larger than that of the conventional materials. One of the reasons for the large dielectric constant of nanocrystalline materials at sufficiently high temperature is the increased space charge polarization due to the structure of their grain boundary interfaces. Also, at sufficiently high temperature the dielectric loss is due to the sharp increase of the dielectric constant at low frequencies and at lower temperatures. As temperature increases, the space charge and ion jump polarization decrease, resulting in a decrease in dielectric constant.

When a dielectric is subjected to an alternating electric field, the electric field strength changes as,

$$
E=E_{0} \cos \omega t
$$

The induced current in the dielectrics does not change exactly with the applied voltage. The current is found to lead the potential in phase. In a similar way, the electrical displacement is also not in phase with respect to E. Now the expression for $\mathrm{D}$ becomes,

$$
\begin{aligned}
& D=D_{0} \cos (\omega t-\delta) \\
& D=D_{0} \cos \omega t \cos \delta+D_{0} \sin \omega t \sin \delta
\end{aligned}
$$

The factor $\sin \delta$ is a measure of the energy absorbed by dielectrics. It is known that in a capacitor the dielectrics usually have a resistance $\mathrm{R}$ and impedance $\mathrm{Z}$ that are related to the phase angle. Assuming $\mathrm{R}$ to be very large,

$$
\sin \delta \approx \tan \delta=1 / \omega R C
$$

The factor $\tan \delta$ is referred to as the dielectric loss. The variations of the dielectric loss of cerium oxide nanoparticles with frequency and temperature are shown in Fig.9. It can be seen that the dielectric loss decreases with increase of frequency and at higher frequencies the loss angle has almost the same value at all temperatures. In dielectric materials, generally dielectric losses occur due to absorption current. The orientation of the molecules along the direction of the applied electric field in polar dielectrics requires a part of the electric energy to overcome the forces of internal friction ${ }^{16}$. One more part of the electric energy is utilized for rotations of dipolar molecules and other kinds of molecular transfer from one position to another, which also involve energy losses. In nanophase materials, inhomogeneities similar to defects and space charge formation in the inter phase layers create an absorption current ensuing in a dielectric loss ${ }^{17}$.

The ac conductivity of the $\mathrm{CeO}_{2}$ nanoparticles, ac conductivity can be calculated by the following relation:

$$
\sigma_{a c}=2 \pi \varepsilon_{0} \varepsilon_{r} f \tan \delta
$$

where $\varepsilon_{0}$ is permittivity in free space, $\varepsilon_{\mathrm{r}}$ is dielectric constant, $\mathrm{f}$ is the frequency and tan $\mathrm{d}$ is the loss factor. The temperature dependent AC electrical conductivity study was also carried out. The temperature dependent AC conductivity of the $\mathrm{CeO}_{2}$ nanoparticles is shown in Fig.10. It is observed that the conductivity increases with increasing frequency. The ac conductivity shows a typical behaviour; a power law increase at frequencies between $10^{5}$ and $10^{7} \mathrm{~Hz}$. This regime is attributed to hopping conduction which sets in frequency above $10^{4} \mathrm{~Hz}$ and the regime is clearly visible for the sample. There is a small increase in the electrical conductivity of the nanomaterial at the low frequency region for an increase in frequency and is the same for all temperatures. Conversely,

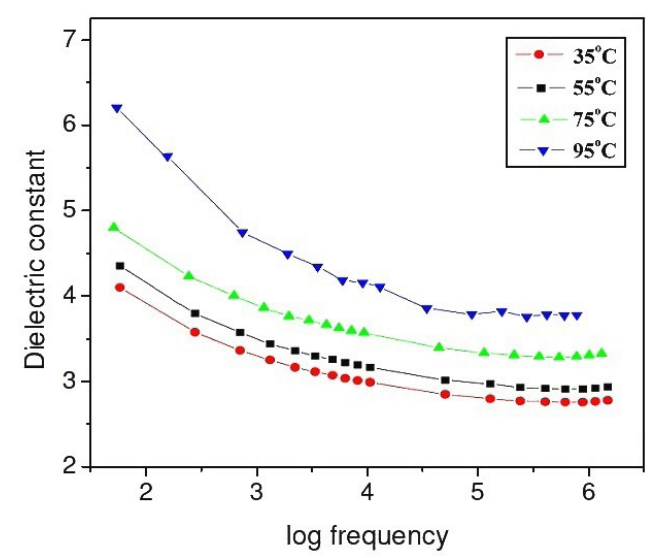

Fig.8. Dielectric constant with log frequency

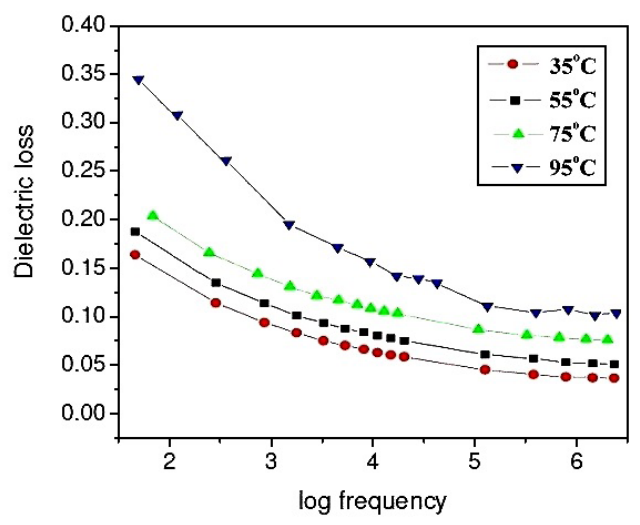

Fig.9. Variation of dielectric loss with $\log$ frequency

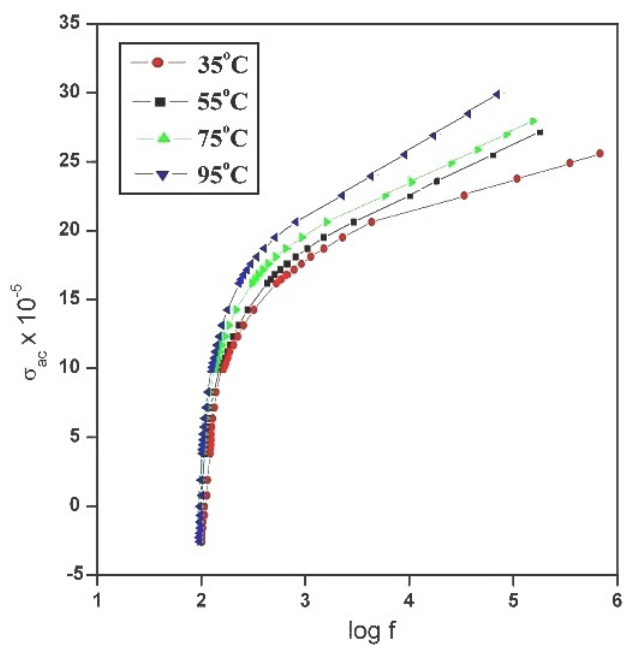

Fig.10. Variation of conductivity with frequency 
at high frequencies especially in the $\mathrm{KHz}$ region, there is an abrupt increase in the conductivity and it is enormous at high temperatures which could be attributed to small polaron hopping.

\section{Conclusions}

Cerium oxide $\left(\mathrm{CeO}_{2}\right)$ nanoparticles were synthesized using simple chemical precipitation method. The formation of $\mathrm{CeO}_{2}$ nanoparticles was confirmed by X-ray diffraction (XRD). The FT-IR spectrum indicated the presence of

\section{References}

1. Guo ZY, Jian FF, Du FL. A simple method to controlled synthesis of $\mathrm{CeO} 2$ hollow microspheres. Scripta Materialia. 2009;61(1):48-51. doi:10.1016/j.scriptamat.2009.03.005

2. Goharshadi EK, Samiee S, Nancarrow P. Fabrication of cerium oxide nanoparticles: Characterization and optical properties. Journal of Colloid \& Interface Science. 2011;356(2):473-480. doi:10.1016/j.jcis.2011.01.063

3. Goharshadi EK, Sara S. Effects of different precursors on size and optical properties of ceria nanoparticles prepared by microwave-assisted method. Materials Research Bulletin. 2012;47(4):1089-1095.

4. Kamruddin M, Ajikumar PK, Nithya R, Raj TB. Synthesis of nanocrystalline ceria by thermal decomposition and softchemistry methods. Scripta Materialia. 2004;50(4):417-422. doi:10.1016/j.scriptamat.2003.11.010

5. Tsai MS. Formation of nanocrystalline cerium oxide and crystal growth. Journal of Crystal Growth. 2005;274(3-4):632-637. doi:10.1016/j.jcrysgro.2004.10.022

6. Lin HC. Production, Application and Market of Cerium Oxide. Hydrometallurgy of China. 2005;24:9-11.

7. Fu Q, Saltsburg H, Flytzani-Stephanopoulos M. Active nonmetallic $\mathrm{Au}$ and Pt species on ceria-based water-gas shift catalysts. Science. 2003;301(5635):935-938.

8. Deluga GA, Salge JR, Schmidt LD, Verykios XE. Renewable hydrogen from ethanol by autothermal reforming. Science. 2004;303(5660):993-997.

9. Hu J, Li Y, Zhou X, Cai M. Preparation and characterization of ceria nanoparticles using crystalline hydrate cerium propionate
$\mathrm{CeO}_{2}$ nanoparticles. Raman spectrum confirmed the cubic nature of the $\mathrm{CeO}_{2}$ nanoparticles. The morphology and the size of the cerium oxide nanoparticles were characterized using Scanning and Transmission Electron Microscopy (SEM and TEM). The optical properties were studied by the UV-Visible absorption spectrum. The variations of the dielectric constant, the dielectric loss with frequency and temperature for $\mathrm{CeO}_{2}$ nanoparticles were analyzed. The $\mathrm{AC}$ electrical conductivity was found to increase with an increase in the temperature and frequency.

as precursor. Materials Letters. 2007;61(28):4989-4992. doi:10.1016/j.matlet.2007.03.097

10. Wang H, Zhu JJ, Zhu JM, Liao XH, Xu S, Ding T, Chen HY. Preparation of nanocrystalline ceria particles by sonochemical and microwave assisted heating methods. Physical Chemistry Chemical Physics. 2002;4(15):3794-3799. DOI: 10.1039/ B201394K

11. Shen G, Wang Q, Wang Z, Chen Y. Hydrothermal synthesis of CeO2 nano-octahedrons. Materials Letters. 2011;65:1211-1214. doi:10.1016/j.matlet.2011.01.057

12. Ketzial JJ, Nesaraj AS. Synthesis of $\mathrm{CeO} 2$ nanoparticles by chemical precipitation and the effect of a sur-factant on the distribution of particle sizes. Journal of Ceramic Processing Research. 2011;12(1):74-79.

13. Dos Santos ML, Lima RC, Riccardi CS, Tranquilin RL, Bueno PR, Varela JÁ, et al. Preparation and characterization of ceria nanospheres by microwave-hydrothermal method. Materials Letters. 2008;62(30):4509-4511. doi:10.1016/j.matlet.2008.08.011

14. Suresh S, Arunseshan C. Dielectric Properties of Cadmium Selenide (CdSe) Nanoparticles synthesized by solvothermal method. Applied Nanoscience. 2014;4(2):179-184.

15. Suresh S. Studies on the dielectric properties of CdS nanoparticles. Applied Nanoscience. 2014;4:325-329.

16. Suresh S. Synthesis and electrical properties of $\mathrm{TiO} 2$ nanoparticles using a wet chemical technique. American Journal of Nanoscience and Nanotechnology. 2013;1(1):27-30. doi: 10.11648/j.nano.20130101.16

17. Suresh S. Synthesis, structural and dielectric properties of zinc sulfide nanoparticles. International Journal of Physical Sciences. 2013;8(21):1121-1127. 